\title{
Estimation of Copper, Silver and Gold Coverages on the Iridium Field Emitter Tip Surface
}

\author{
Khalil Ismaiel Hashim \\ Physics Department, Faculty of Science, Benghazi University, Benghazi, Libya \\ Email: khalilismaiel@gmail.com
}

How to cite this paper: Hashim, K.I. (2018) Estimation of Copper, Silver and Gold Coverages on the Iridium Field Emitter Tip Surface. World Journal of Engineering and Technology, 6, 556-566. https://doi.org/10.4236/wjet.2018.63033

Received: June 12, 2018

Accepted: July 6, 2018

Published: July 9, 2018

Copyright $\odot 2018$ by author and Scientific Research Publishing Inc. This work is licensed under the Creative Commons Attribution International License (CC BY 4.0).

http://creativecommons.org/licenses/by/4.0/

\section{cc (i) Open Access}

\begin{abstract}
The work described in this paper is a study of the estimation of copper, silver and gold coverages on the iridium field emitter tip surface. The study has been carried out by using a simple field emission microscope designed especially for the purpose of the adsorbate coverage calibration. It was equipped with an iridium field emitter tip. On one side of the microscope was the vapor source $12.5 \mathrm{~cm}$ from the tip, and on the other side $16.2 \mathrm{~cm}$ from the source was a quartz crystal oscillator. The crystal leads were spot welded to a two-pin tungsten-glass press-seal. In front of the crystal, a nickel shield was mounted in which there was a circular hole of an area of $0.0804 \mathrm{~cm}^{2}$, slightly smaller than the surface of the crystal, to prevent shorting of the conducting ends of the crystal which would be brought about by the condensed metal. The sensing crystal inside the microscope was driven by a small circuit placed just outside the microscope. The driving circuit was in turn connected to another circuit which comprised a frequency comparator unit which could read the frequency of the quartz crystal oscillator before and after the deposition of the adsorbate and gave a direct digital reading of $\frac{\Delta f}{f}$ ( $f$ is the resonance frequency of the crystal before the deposition of the adsorbate and $\Delta f$ is the difference in the frequency of the oscillator after and before the deposition of the adsorbate on the crystal). The mass added to either side of the crystal alters its resonant frequency. The frequency shift obtained for a certain thickness of the deposited film depends on the density of the deposited film [1] [2].
\end{abstract}

\section{Keywords}

Field Emission Microscope, Electron Work Function, Adsorbate Coverage Calibration, A Quartz Crystal Oscillator, Resonant Frequency, Fundamental Frequency, A Frequency Comparator Unit, Deposition, Monolayer 


\section{Introduction}

Copper, silver and gold vapors were deposited on the tip surface in doses; every dose was obtained by heating the source resistively for a fixed duration of time. The number of atoms of the studied materials deposited on the tip surface is needed to be known. This has been measured in this study using a quartz crystal microbalance.

The microbalance operates on the principle that when a material is deposited on the oscillating surface of a quartz crystal, its frequency changes and the amount of the frequency shift is proportional to the mass deposited and hence to the number of deposited atoms. The frequency shift is measured and the number of the deposited atoms can be computed using some equations relating them to the frequency shift.

\section{Theory}

The fundamental frequency of an AT cut crystal is given by [3]

$$
f=\frac{1}{2 d}\left(\frac{q}{p}\right)^{\frac{1}{2}}
$$

where $d$ is the thickness of the crystal, $p$ its density, and $q$ the stiffness factor.

The density $p$ can be written as

$$
P=\frac{M}{V}
$$

where $M$ is the mass of the crystal and $V$ the vibrating volume which can be written as

$$
V=A \cdot d
$$

where $A$ is the vibrating area of the crystal. Combining Equations (2) and (3), $d$ can be written as:

$$
d=\frac{M}{p A}
$$

Substituting Equation (4) into Equation (1) gives

$$
f=\frac{p A}{2 M}\left(\frac{q}{p}\right)^{\frac{1}{2}}=\frac{A}{2 M}(p q)^{\frac{1}{2}}
$$

Differentiating Equation (5) with respect to $M$ gives

$$
\frac{\mathrm{d} f}{\mathrm{~d} M}=-\frac{A}{2 M^{2}}(p q)^{\frac{1}{2}}
$$

From Equations (5) and (6)

$$
\frac{\mathrm{d} f}{\mathrm{~d} M}=-\frac{f}{M} \rightarrow \frac{\mathrm{d} f}{f}=-\frac{\mathrm{d} M}{M}
$$

Equation (7) shows that the change in the resonant frequency $\mathrm{d} f$ is proportional to the increase in the mass, $\mathrm{d} M$, deposited on the crystal surface. The neg- 
ative sign implies a decrease in the frequency.

The statement $\mathrm{d} f$ is proportional to $\mathrm{d} M$ is valid only for films which are sufficiently thin compared with the thickness of the quartz crystal [1]. Thus the limitation $\frac{\mathrm{d} M_{\text {dep }}}{M} \ll 1$ must be applied [4].

\section{The Advantages and Disadvantages of the Quartz Crystal Microbalance}

The advantages of using this technique to estimate the adsorbate coverage on the substrate surface outweigh the disadvantages. The advantages are:

1) The quartz crystal is bakeable at high temperature and then compatible with ultra high vacuum processing. (We baked it to a temperature which was as high as $425^{\circ} \mathrm{C}$.)

2) The measurement is fast.

3) It is not limited by material.

4) It is highly sensitive even to a very small deposit, $\approx 10^{-9} \mathrm{~g}[1]$.

The disadvantages are:

1) The sticking probability of the depositing material may be different on the quartz crystal plate from that on the substrate in use.

2) It is heat sensitive.

\section{The Experimental Arrangement}

The experimental arrangement in the present study consisted, as shown in Figure 1, of a simple field emission microscope designed especially for the purpose of the adsorbate coverage calibration. It was equipped with an iridium field emitter tip. On one side of the microscope was the vapor source $12.5 \mathrm{~cm}$ from the tip, and on the other side $16.2 \mathrm{~cm}$ from the source was an AT cut quartz crystal oscillator as shown in Figure 2. The crystal leads were spot welded to a two-pin tungsten-glass press-seal. In front of the crystal a nickel shield was mounted in which there was a circular hole of area of $0.0804 \mathrm{~cm}^{2}$, slightly smaller than the surface of the crystal, to prevent shorting of the conducting ends of the crystal which would be brought about by the condensed metal.

The sensing crystal inside the microscope was driven by a small circuit placed just outside the microscope. The driving circuit was in turn connected to another circuit which comprised a frequency comparator unit which could read the frequency of the quartz crystal oscillator before and after the deposition of the adsorbate and gave a direct digital reading of $\frac{\Delta f}{f}$ ( $f$ is the resonance frequency of the crystal before the deposition of the adsorbate and $\Delta f$ is the difference in the frequency of the oscillator after and before the deposition of the adsorbate on the crystal).

The mass added to either side of the crystal alters its resonant frequency. The frequency shift obtained for a certain thickness of the deposited film depends on the density of the deposited film [1] [2]. 


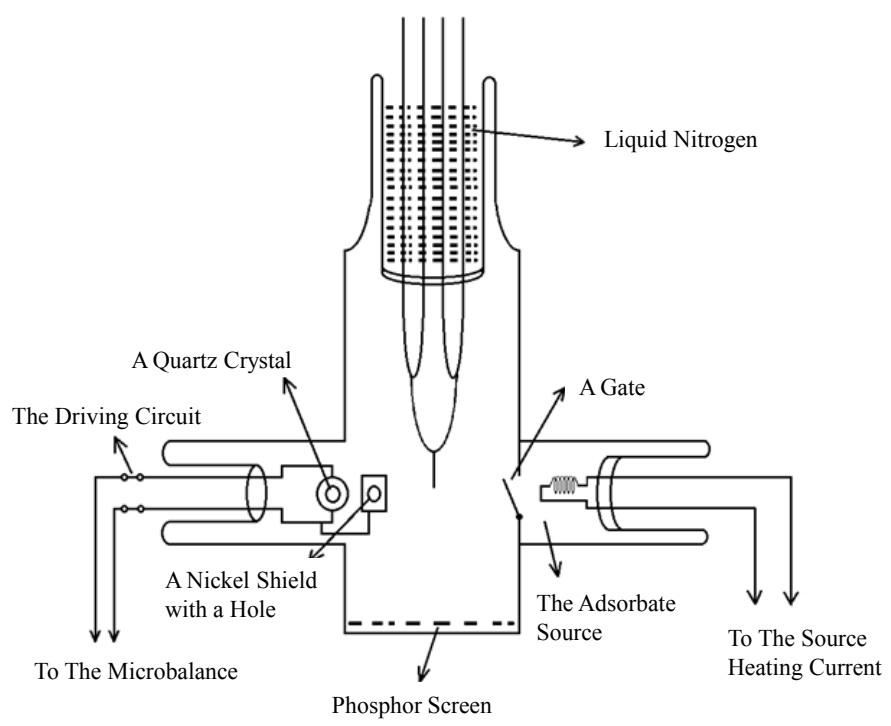

Figure 1. A schematic diagram of the experimental arrangement used for measuring the amount of the adsorbate.

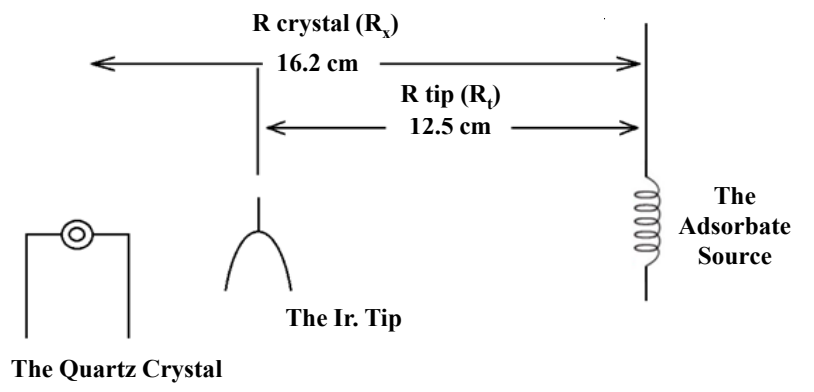

Figure 2. The distances between the source and he tip and between the source and the quartz crystal.

The vapor source to be calibrated was evaporated simultaneously on the tip which was kept at liquid nitrogen temperature and onto the quartz crystal which was kept at room temperature.

The source was heated while the gate in front of the source (see Figure 1) was closed and after about one minute the gate was opened to allow the vapor to condense on the tip surface. After three minutes the gate was closed and the deposit uniformly diffused over the tip surface. This was repeated until the average work function for copper, silver and gold deposits attained their coverage-Independent values, $\varnothing_{\text {sat }}$.

\section{Results and Discussion}

The time, $t_{s}$ taken just to attain $\varnothing_{\text {sat }}$ for each vapor source was measured (points A, B, and C on Figures 3-5 for copper, silver and gold respectively).

The source was then heated and the vapor was allowed to deposit on the tip and quartz crystal continuously for the measured time, $t_{s}$, and the resulting value of $\frac{\Delta f}{f}$ was recorded. 
The sticking probabilities of copper, silver and gold on the quartz crystal at room temperature and on tip at liquid nitrogen temperature were assumed to be the same and equal to unity [5] [6] [7].

In order to ensure that the measured $\frac{\Delta f}{f}$ contained no spurious thermal effects it was proved necessary to allow the crystal to cool down for a period between a half of an hour and one hour.

Due to the difference in the distance of the crystal and the tip relative to the source (Figure 2) the atomic concentration at the tip will differ from that on the crystal, and according to the inverse square law, the atoms concentration at a point is inversely proportional to the square of the distance of that point from the metal source.

When $R_{x}$ is the distance from source-to-crystal, and $R_{t}$ is the distance from source-to-tip, then

$$
\frac{\text { Atomic Concentration on the tip }}{\text { Atomic Concentation on the crystal }}=\frac{R_{x^{2}}}{R_{t^{2}}}
$$

In our study, $R_{x}$ and $R_{t}$ were $16.2 \mathrm{~cm}, 12.5 \mathrm{~cm}$ respectively, therefore

$$
\frac{R_{x^{2}}}{R_{t^{2}}}=1.68
$$

The obtained $\frac{\Delta f}{f}$ for copper coverage at $\varnothing_{\text {sat }}$ was found to be $0.0000028 \pm$ 0.0000004 , where the 0.0000004 is the accuracy of the outputted value.

Taking $\frac{\Delta f}{f}$ to be only 0.0000028 and using Equation (7), the amount of copper deposited on the crystal surface was

$$
\Delta M=6.44 \times 10^{-8} \mathrm{gm}\left(M_{\text {crystal }}=0.023 \mathrm{gm}\right)
$$

The density of the deposited copper $\left(P_{c u}\right)$ on the crystal surface was found by dividing $\Delta M$ by the area of the nickel hole in front of the crystal $(A=0.0804$ $\mathrm{cm}^{2}$ ), thus

$$
P_{c u}=\frac{6.44 \times 10^{-6} \mathrm{gm}}{0.0804 \mathrm{~cm}^{2}}=8.0074 \times 10^{-7} \mathrm{gm} \cdot \mathrm{cm}^{-2}
$$

From Equation (8) the density of copper on the tip surface was found to be $1.3452 \times 10^{-6} \mathrm{gm} \cdot \mathrm{cm}^{-2}$.

The density of the deposit condensed on the side of an effectively cylindrical shank is attenuated by a factor of $1 / \pi$ on spreading over the entire emitting area [8]. Therefore the true density of copper on the tip surface would be

$$
1.3452 \times 10^{-6} \div \pi=4.282 \times 10^{-7} \mathrm{gm} \cdot \mathrm{cm}^{-2}
$$

The atomic weight of copper is 63. One gram atom of copper therefore weights $63 \mathrm{gms}$ and one gram atom contains $A_{v}$ atoms where $A_{v}$ is Avogadro's 
number and equal to $6.023 \times 10^{23}$ atoms per mole.

Therefore 63 gms of copper contain $A_{V}$ number of atoms. The density of copper in $\mathrm{gms} \cdot \mathrm{cm}^{-2}$ at $\varnothing_{\text {sat }}$ is equivalent to

$$
\frac{6.023 \times 10^{23}}{63} \times 4.282 \times 10^{-7} \text { atom } \cdot \mathrm{cm}^{-2}=4.0938 \times 10^{15} \text { atom } \cdot \mathrm{cm}^{-2}
$$

Taking into account the \pm 0.0000004 in $\frac{\Delta f}{f}$, the copper concentration at $\Phi_{\text {sat }}$ would be $\approx(4.1 \pm 0.5) \times 10^{15}$ atoms $\cdot \mathrm{cm}^{-2}$.

Assuming a mean surface atom density of $1 \times 10^{15}$ atoms $\cdot \mathrm{cm}^{-2}$ on the iridium points, the saturation coverage of copper on iridium occurred at a coverage of $\theta$ $=4.1 \pm 0.5$, where $\theta=1$ is one monolayer and defined as [9].

$\frac{\text { Mean density of copper atoms on iridium surface }}{\text { Mean density of iridium atoms on the surface }}$

Following the same procedure, the calculated saturation coverages of silver (at $\left.\varnothing_{\text {sat }}\right)$ and gold occurred at $\theta=5.25 \pm 0.25$ and $\theta=2.25 \pm 0.18$ respectively.

Amounts of copper, silver and gold condensed on the tip at different fluxes have been measured as fractions of the coverage taken to reach $\Phi_{\text {sat }}$.

\section{Coverage Criteria}

Changes of the work function of metals due to the adsorption of atoms on their surfaces depend on the charge of the adsorbed atoms with respect to that of the substrate metal. Generally electropositive atoms adsorbed on the substrate metal form systems with lower work function than that of the substrate [8] [10] [11] [12] [13] [14], and electronegative atoms form systems with higher work function than that of the substrate [5] [9] [15]-[25]. However, some adsorbates behave differently on different planes of the same surface depending on the structure and electronegativity of the plane in question [18] [19] [23] [26] [27] [28].

In the present study copper and silver invariably decrease the average work function when adsorbed on the iridium surface while gold either increases or decreases the average work function depending on the spreading temperature. Thus copper and silver behave as if positively charged when adsorbed on iridium and this contrasts with their behavior when adsorbed on tungsten [9] [15] [19] [21] [29]. Gold on the other hand, behaves as positively charged under some conditions and negatively charged under others when adsorbed on iridium while it is always negatively charged with respect to tungsten [5] [18] [29].

Other changes in the work function take place at particular concentrations of the adsorbates, and in order to interpret these changes, an accurate knowledge of adatom coverage is necessary. A convenient measure of coverage is the monolayer which is denoted by $\theta=1$ and defined by

Mean density of the dsorbate atoms on substrate surface

Mean density of the substrate atoms on the substrate surface 
In most cases when the adsorbates increase the work function of the substrate when adsorbed on its surface and the work function passes through a maximum $\Phi_{\text {maxt }}$, the coverage at that $\Phi_{\max }$, is considered to be at or close to $\theta=1$. However, [30] considers a monolayer coverage to be one at which the work function is minimum.

Figures 3-5 show that the measured average coverages of copper, silver, and gold on iridium substrate surface at $\Phi_{\text {sat }}$ are $\theta=4, \theta=5.33$, and $\theta=2.1$ respectively, where $\theta=1$ is one layer of the adsorbate on the substrate.

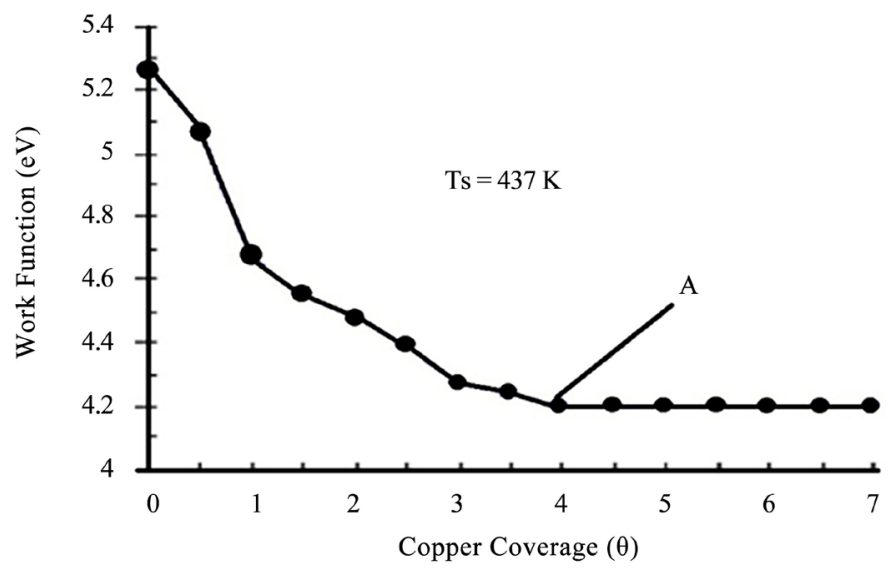

Figure 3. Change in work function with copper coverage.

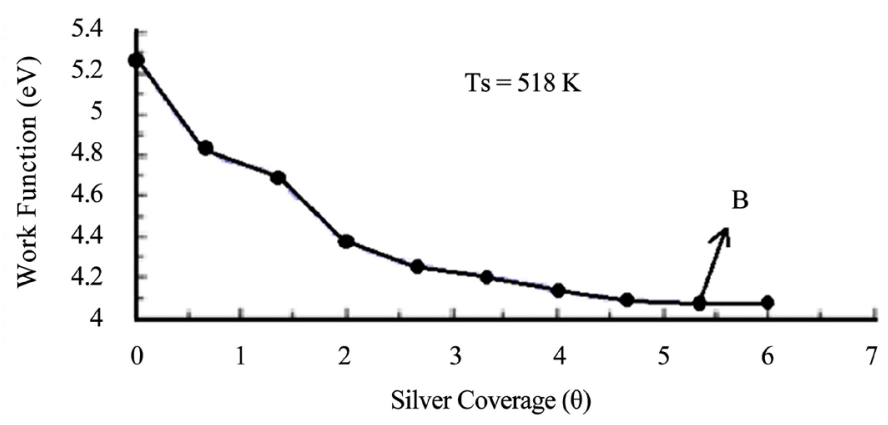

Figure 4. Change in work function with silver coverage.

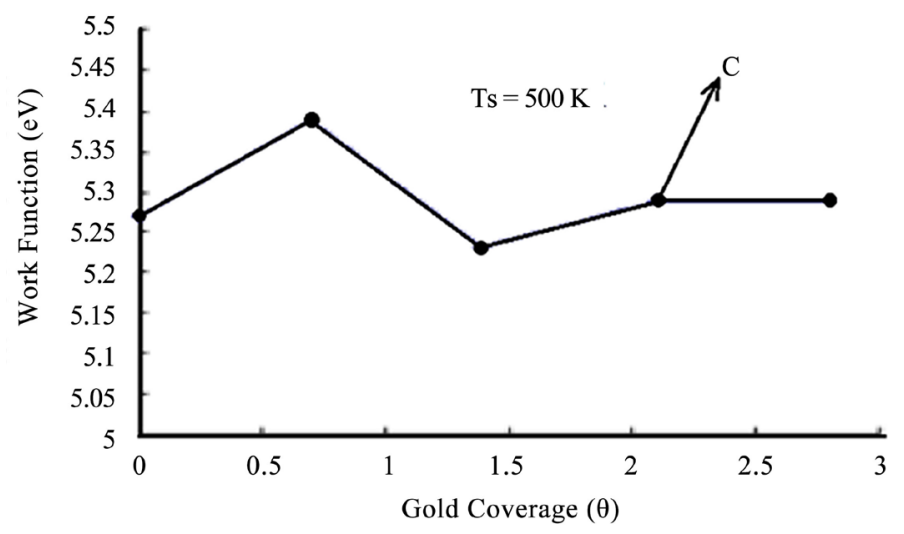

Figure 5. Change in work function with gold coverage. 
In his measurements, [5] found the concentration of gold on tungsten at $\Phi_{\max }$ to be $6.7 \times 10^{18}$ atoms $\cdot \mathrm{m}^{-2}$, and the average concentration of surface tungsten atoms is $5.7 \times 10^{18}$ atoms $\cdot \mathrm{m}^{-2}$, this gives a gold: tungsten atom ratio of 1.17 , a value to that expected for the monolayer as defined. This view is supported by [31] using field ion microscopy. However in some cases the calculated value for the coverage at $\Phi_{\max }$ is not $\theta=1$ [9] [21].

Another criteria for $\theta=1$ is that of the coverage at which a pseudoclean emission pattern is formed [8]. Our observations for copper, silver, and gold adsorption on iridium substrate show that this criterion is not met because we observed that at no coverage of these adsorbates did the emission patterns depart substantially from that of clean iridium. The similarity between patterns produced by adsorbed copper, silver, and gold and that formed by clean iridium suggests that the long-range morphology of the adsorbate layers is determined the structure of the iridium substrate. [32] developed and performed a statistical study on the method of a coverage estimation. A coverage prediction based on FEM-DEM $($ DME $=$ Discrete Element Model, FEM = Finite Element Method $)$ simulation was developed and validated by [33]. The numerical coverage study based on FEM-DEM method exhibited the same trend as the experimental data. It is shown that the resonance frequency (f) and quality factor (Q) of the resonator depend on the existence and the type of the monolayer [34]. [35] used a quartz crystal microbalance to monitor the adsorption of biomolecules to the resonator surface, the fractional coverage of the surface, $\theta$ was correlated with the frequency shift of the resonator, $\Delta \mathrm{f}$. The adsorbed material was ferritin, which is a spherical protein with a diameter of $\approx 12 \mathrm{~nm}$. [36] in their study investigated the deposition of monodisperse, amine functionalized silica nanoparticles on gold surfaces using quartz crystal microbalance (QCM) to obtain frequency and dissipation changes during adsorption from the liquid phase. [37] observed positive shifts of frequency, $\Delta \mathrm{f}$, in contrast to the negative frequency shifts typically found in adsorption experiments. A comparison of frequencies shifts and bandwidths on different overtones reveals a coupled resonance: at low overtones, $\Delta \mathrm{f}$ is negative, whereas it is positive at high overtones [37].

\section{Conclusions}

Changes in the work function of the substrate due to the adsorption of the adsorbate atoms take place at different concentrations of the adsorbates, and in order to interpret these changes, an accurate knowledge of adatom coverage is necessary. In most cases when the adsorbates increase the work function of the substrate when adsorbed on its surface and the work function passes through a maximum $\left(\Phi_{\max }\right)$, the coverage at that $\Phi_{\max }$, is considered to be at or close to $\theta=$ 1. However, [30] considers a monolayer coverage to be one at which the work function is minimum.

The microbalance operates on the principle that when a material is deposited on the oscillating surface of a quartz crystal, its frequency changes and the amount of the frequency shift is proportional to the mass deposited and hence to 
the number of deposited atoms. The frequency shift is measured and the number of the deposited atoms can be computed using some equations relating them to the frequency shift.

In order to ensure that the measured $\frac{\Delta f}{f}$ contained no spurious thermal effects, it was proved necessary to allow the crystal to cool down for a period between a half of an hour and one hour.

The saturation coverage of copper on iridium occurred at a coverage of $\theta=4.1 \pm$ 0.5 , where $\theta=1$ is one monolayer, while the calculated saturation coverages of silver at $\Phi_{\text {sat }}$ and gold occurred at $\theta=5.25 \pm 0.25$ and $\theta=2.25 \pm 0.18$ respectively.

\section{References}

[1] Webby, G.M. (1985) Honours Dissertation. University College of North Wales, Bangor.

[2] Sangar, C.C. (1968) Honours Dissertation. University College of North Wales, Bangor.

[3] Roberts, E.W. (1971) Honours Dissertation. University College of North Wales, Bangor.

[4] Pulker, H.K. (1968) Factors Influencing the Accuracy of a Quartz-Crystal-Oscillator as a Thickness Monitor for Thin Film Deposition. Thin Solid Films, 1, 400-402. https://doi.org/10.1016/0040-6090(68)90032-1

[5] Jones, J.P. and Jones, N.T. (1967) Surface Phenomena of Metals, Monograph No. 28, Society of Chemical Industry, London, 263.

[6] Cetronio, A., Jones, J.P. and Roberts, E.W. (1975) A Study of Copper Adsorption on (100) Tungsten by High Field Microscopy. Surface Science, 52, 473-488. https://doi.org/10.1016/0039-6028(75)90082-5

[7] Smith, G.D.W. (1973) Pseudomorphism in Ultra-Thin Metal Films. Surface Science, 35, 304-318 https://doi.org/10.1016/0039-6028(73)90221-5

[8] Schmidt, L. and Gomer, R. (1965) Adsorption of Potassium on Tungsten. Journal of Chemical Physics, 42, 3573. https://doi.org/10.1063/1.1695764

[9] Jones, J.P. (1972) Adsorption of Silver on Tungsten. Surface Science, 32, 29-44. https://doi.org/10.1016/0039-6028(72)90119-7

[10] Sidorski, Z., Pelly, I. and Gomer. R. (1969) Adsorption of Cs on Tungsten: Measurements on Single-Crystal Planes. Journal of Chemical Physics, 50, 2382-2391. https://doi.org/10.1063/1.1671392

[11] Al-Rawi, O.Z. (1980) Adsorption of Silver on Rhenium by Field Emission Microscopy. PhD Thesis, University College of North Wales, Bangor.

[12] Anderson, J., Danforth, W.E. and Williams, A.J. (1963) Work Function and Thermal Desorption in the System Thorium-on-Rhenium. Journal of Applied Physics, 34, 2260-2265. https://doi.org/10.1063/1.1702725

[13] Al-Rawi, O.Z. and Jones, J.P. (1983) A Field Emission Study of Silver on Rhenium. Surface Science, 124, 220-240. https://doi.org/10.1016/0039-6028(83)90346-1

[14] Haynes, A.S. (1987) Honours Dissertation. University College of North Wales, Bangor.

[15] Jones, J.P. (1965) The Adsorption of Copper on Tungsten. Proc. Roy. Soc., London, Vol. A 284, 469-487. https://doi.org/10.1098/rspa.1965.0075 
[16] Jones, N.T. (1971) Adsorption of Gold on Tungsten by Field Emission Microscopy. PhD Thesis, University College of North Wales, Bangor.

[17] Gorodetskii, V.V., Sobyanin, V.A., Bulgakov, N.N. and Knor, Z. (1979) Low Temperature Reaction of Hydrogen with Preadsorbed Oxygen on Iridium Surfaces. Surface Science, 82, 120-138. https://doi.org/10.1016/0039-6028(79)90323-6

[18] Jones, J.P. and Jones, N.T. (1976) Field Emission Microscopy of Gold on Single-Crystal Planes of Tungsten. Thin Solid Films, 35, 83-97. https://doi.org/10.1016/0040-6090(76)90243-1

[19] Polanski, J. and Sidorski, Z. (1973) Adsorption of Copper on Tungsten; Measurements on Single Crystal Planes. Surface Science, 40, 282-294. https://doi.org/10.1016/0039-6028(73)90068-X

[20] Coles, S.J.T. and Jones, J.P. (1977) Adsorption of Gold on Low Index Planes of Rhenium. Surface Science, 68, 312-327. https://doi.org/10.1016/0039-6028(77)90217-5

[21] Sugata, E. and Takeda, K. (1970) Adsorption and Nucleation of Silver on Tungsten. Physica Status Solidi, 38, 549. https://doi.org/10.1002/pssb.19700380205

[22] Lewis, R. and Gomer, R. (1968) Adsorption of Oxygen on Platinum. Surface Science, 12, 157-176. https://doi.org/10.1016/0039-6028(68)90121-0

[23] Jones, G. (1987) Honours Dissertation. University College of North Wales, Bangor.

[24] Bell, A.A. and Gomer, R. (1966) Adsorption of Carbon Monoxide on Tungsten. Abundances, Dipole Moments, and Sticking Coefficients. The Journal of Chemical Physics, 44, 1065-1080. https://doi.org/10.1063/1.1726790

[25] Bell, A.E., Swanson, L.W. and Crouser (1968) A Field Emission Study of Oxygen Adsorption on the (110), (211), (111) and (100) Planes of Tungsten. Surface Science, 10, 254-274. https://doi.org/10.1016/0039-6028(68)90023-X

[26] Delchar, T.A. and Ehrlich, G. (1965) Chemisorption on Single Crystal Planes: Nitrogen on Tungsten. The Journal of Chemical Physics, 42, 2686.

https://doi.org/10.1063/1.1703224

[27] Roberts, E.W. (1974) Adsorption of Copper on Tungsten. PhD Thesis, University College of North Wales, Bangor.

[28] Jowett, C.W. and Hopkins, B.J. (1970) Work Function Changes Due to the Adsorption of Chlorine, Bromine and Iodine on Tungsten Single Crystal Surfaces. Surface Science, 22, 392-410. https://doi.org/10.1016/0039-6028(70)90090-7

[29] Cetronio, A. and Jones, J.P. (1974) A Study by High Field Microscopy of the Effect of Substrate Surface Structure on the Work Function of Layers of Group 1b Metals Adsorbed on Tungsten. Surface Science, 44, 109-128. https://doi.org/10.1016/0039-6028(74)90096-X

[30] Marinova, Ts.S. and Zubenko, Tu.V. (1970) Soviet Physics. Solid State, 12, 398-400.

[31] Martin, A.D. (1970) Field Desorption of Gold Layers from Tungsten. PhD Thesis, University College of North Wales, Bangor.

[32] Bagherifard, S., Ghelichi, R. and Guagliano, M. (2012) On the Shot Peening Surface Coverage and Its Assessment by Means of Finite Element Simulation: A Critical Review and Some Original Developments. Applied Surface Science, 259, 186-194. https://doi.org/10.1016/j.apsusc.2012.07.017

[33] Zhang, J., Lu, S., Wu, T., Zhou, Z. and Zhang, W. (2018) An Evaluation on SP Surface Property by Means of Combined FEM-DEM Shot Dynamics Simulation. Advances in Engineering Software, 115, 283-296. https://doi.org/10.1016/j.advengsoft.2017.10.001

[34] Zarifi, M.H., Farsinezhad, S., Wiltshire, B.D., Abdorrazaghi, M., Mahdi, N., Kar, P., 
Daneshmand, M. and Shankar, K. (2016) Effect of Phosphonate Monolayer Adsorbate on the Microwave Photoresponse of TiO Nanotube Membranes Mounted on a Planar Double Ring Resonator. Nanotechnology, 27, Article ID: 375201. https://doi.org/10.1088/0957-4484/27/37/375201

[35] Johannsmann, D., Reviakine, I. and Richter, R.P. (2009) Dissipation in Films of Adsorbed Nanospheres Studied by Quartz Crystal Microbalance (QCM). Analytical Chemistry, 81, 8167-8176. https://doi.org/10.1021/ac901381z

[36] Grunewald, C., Schmudde, M., Noufele, C.N., Graf, C. and Risse, T. (2015) Ordered Structures of Functionalized Silica Nanoparticles on Gold Surfaces: Correlation of Quartz Crystal Microbalance with Structural Characterization. Analytical Chemistry, 87, 10642-10649. https://doi.org/10.1021/acs.analchem.5b03572

[37] Pomorska, A., Shchukin, D., Hammond, R., Cooper, M.A., Grundmeier, G. and Johannsmann, D. (2010) Positive Frequency Shifts Observed upon Adsorbing Micron-Sized Solid Objects to a Quartz Crystal Microbalance from the Liquid Phase. Analytical Chemistry, 82, 2237-2242. https://doi.org/10.1021/ac902012e 